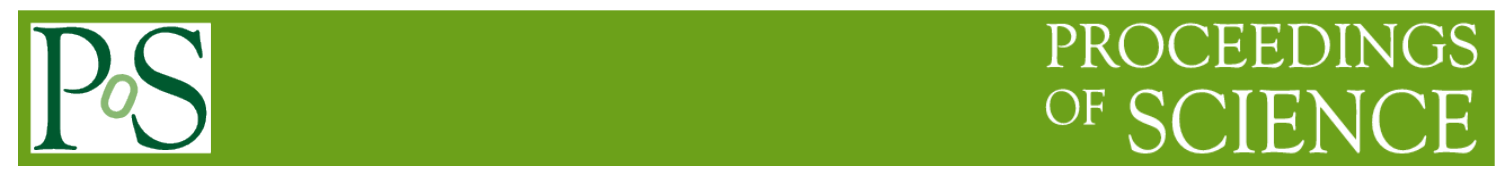

\title{
Very Long Baseline Interferometry: the EVN, WSRT, and JIVE
}

\section{Huib Jan van Langevelde}

Joint Institute for VLBI ERIC

Oude Hoogeveensedijk 4, 7991PD Dwingeloo, The Netherlands

E-mail: langevelde@jive.eu

\section{Richard Schilizzi}

School of Phys. \& ASTRON, Alan Turing Bldg., University of Manchester, Oxford Road, Manchester M13 9PL, UK

E-mail: richard.schilizzi@manchester.ac.uk

\section{Arnold van Ardenne}

ASTRON

Oude Hoogeveensedijk 4, 7991 PD Dwingeloo, The Netherlands

E-mail: ardenne@astron.nl

\section{Lout Sondaar $\dagger$}

ASTRON

\section{Albert Jan Boonstra \\ ASTRON \\ Oude Hoogeveensedijk 4, 7991 PD Dwingeloo, The Netherlands \\ E-mail: boonstra@astron.nl}

50 Years Westerbork Radio Observatory, A Continuing Journey to Discoveries and Innovations Richard Strom, Arnold van Ardenne, Steve Torchinsky (eds) 


\section{Chapter 7 Very Long Baseline Interferometry: the EVN, WSRT, and JIVE}

Huib Jan van Langevelde', Richard Schilizzi², Arnold van Ardenne ${ }^{3}$

\section{Introduction}

Very-long-baseline interferometry (VLBI) is the technique used to study small radio sources using an array of telescopes with separations typically ranging from a few $100 \mathrm{~km}$ to many thousands of $\mathrm{km}$, including telescopes in Earth orbit. Such an array can be either a dedicated array of telescopes like the US VLB Array or an array of existing national telescopes like the EVN. The network elements must all observe the same target at the same time and with the same frequency sampling, in order to allow their data to be correlated in a central data processor for all telescope pairs. The resulting 'fringe visibilities' can be processed by astronomers to yield high angular resolution images of the astronomical targets. Until the advent of fibre links between telescopes, each telescope operated independently under the control of an atomic oscillator providing frequency and time stability, recording the incoming signals on tape for later shipment to a central location for data processing.

This chapter will take a brief look at the development of VLBI in the Netherlands including use of the WSRT and the gradually emerging leadership role in European and global VLBI undertaken by the NFRA, later ASTRON, and the Joint Institute for VLBI in Europe (JIVE), later JIVE ERIC. It will also briefly review a number of technical developments including the WSRT-specific instrumentation developed for the WSRT as a VLBI element, the EVN MkIV correlator at JIVE, VLBI using optical fibre networks, and the use of the WSRT as a test platform for advancing the VLBI technique for world VLBI. Finally we review some of the major scientific results resulting from VLBI observations

Joint Institute for VLBI in Europe ERIC, Sterrewacht Leiden, Leiden University, The Netherlands ${ }^{2}$ University of Manchester, UK ${ }^{3}$ ASTRON , The Netherlands using the WSRT as one of the elements, and conclude with some observations on the future of this subject.

\subsection{A brief history of VLBI in the Netherlands}

To put the VLBI developments in the Netherlands in context, Table 1 lists the major events in international VLBI as seen from a Dwingeloo perspective (see also [1] [2]). 
Table 1: Major events in the development of VLBI, from a Dwingeloo perspective

\begin{tabular}{|c|c|c|c|}
\hline 1967 & first VLBI observations, in Canada and the USA & 1982 & $\begin{array}{l}\text { MPIfR MkIII tape recorder-based data } \\
\text { processor starts operation }\end{array}$ \\
\hline 1968 & first US-Europe (Sweden) observations & 1993 & $\begin{array}{l}\text { JIVE established as a Dutch Scientific } \\
\text { Foundation in Dwingeloo }\end{array}$ \\
\hline 1975 & first discussions of European VLBI & & US VLB Array opened \\
\hline \multirow[t]{2}{*}{1976} & US VLBI Network formed & 1997 & $\begin{array}{l}\text { Japanese space VLBI telescope, VSOP-HALCA, } \\
\text { launched }\end{array}$ \\
\hline & $\begin{array}{l}\text { first 3-station intra-European VLBI } \\
\text { observations }\end{array}$ & 1998 & JIVE Data Processor opened \\
\hline \multirow[t]{2}{*}{1978} & $\begin{array}{l}\text { first VLBI observations with WSRT in tied-array } \\
\text { mode }\end{array}$ & 2004 & $\begin{array}{l}\text { First e-VLBI observations using optical fibre } \\
\text { connections between telescopes and data } \\
\text { processor at JIVE }\end{array}$ \\
\hline & $\begin{array}{l}\text { MPIfR MkII tape recorder-based data } \\
\text { processor starts operation }\end{array}$ & 2011 & $\begin{array}{l}\text { Russian space VLBI telescope, RadioAstron, } \\
\text { launched }\end{array}$ \\
\hline 1980 & European VLBI Network formed & 2015 & JIVE becomes a European Legal Entity (ERIC) \\
\hline
\end{tabular}

(U) an VLBI network involved George Miley (ULeiden), Roy Booth (UManchester, later Onsala Space Observatory), and Ivan Pauliny-Toth (Max-Planck Institute for Radio astronomy, MPIfR) and took place in the MPIfR cafeteria in Bonn, West Germany. Dutch participation in a second meeting a few months later in Bonn expanded to include Boudewijn Baud, Harm Habing and George Miley from Leiden, and Wim Brouw and Jean Casse from SRZM in addition to a larger delegation from Germany. Early in 1976, a VLBI recording system was ordered for the Dwingeloo 25m telescope and plans were laid by Miley, Habing, Baud and Schilizzi for the first 3-station intra-European observations involving Dwingeloo, Onsala and Effelsberg (Figure 1). These were successful and led to a paper on the compact radio structure in the centre of the largest known extragalactic radio source, ${ }_{3} \mathrm{C}_{23} 6[3]$. This signalled the beginning of a new and

Figure 1. 1976 Dwingeloo $18 \mathrm{~cm}$ observation inside the telescope pedes tal (!) with Richard Schilizzi and George Miley actively adjusting the recording terminal on loan from the Onsala Space Observatory

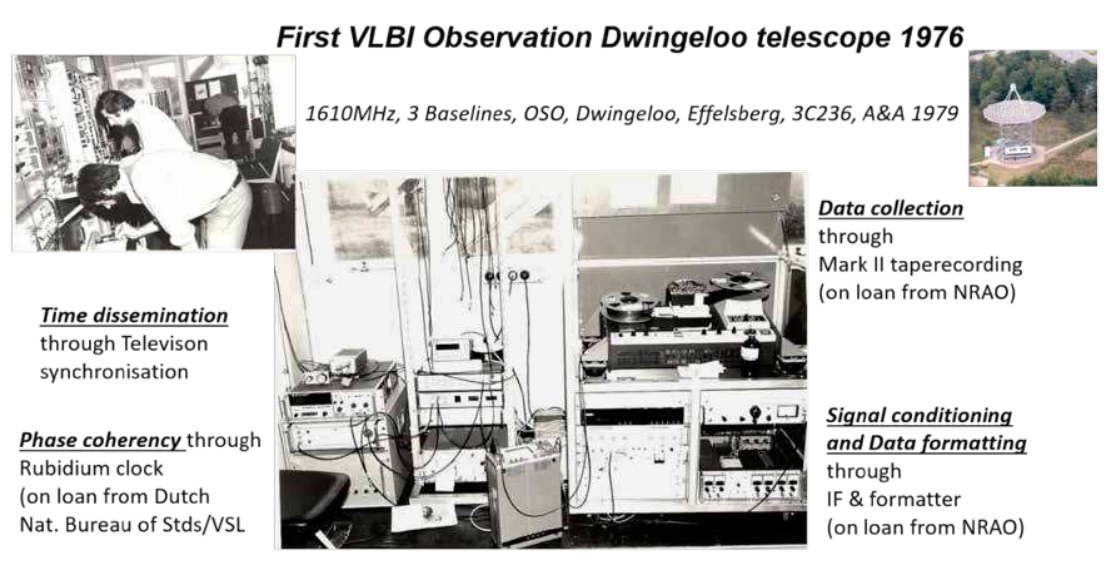

exciting strand of astronomical research in the Netherlands whose prospects were outlined in [4]

One of those prospects, much increased VLBI sensitivity, quickly led to plans to phase up the WSRT to create the equivalent of a single telescope with a $93 \mathrm{~m}$ di. Arnold van Ardenne succeeded in designing a first generation tied-array backend in 1978 ; a photograph of first fringes as they appeared on the screen of the MPIfR MkII data processor is shown in Figure 2. The tied array design and its evolution are described in Section 2.1

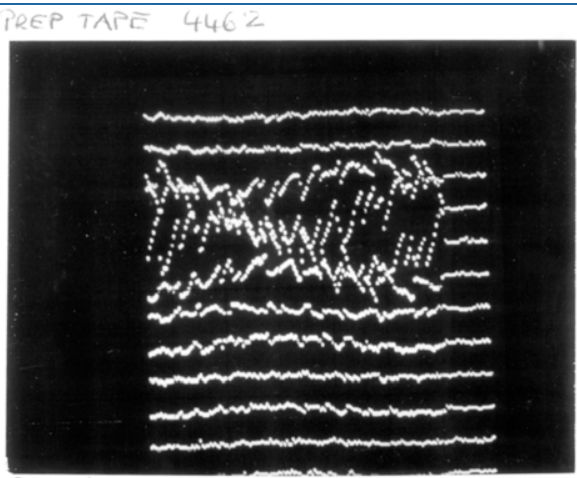

Figure 2. First interference fringes on the WSRT-Effelsberg baseline in 1978. The plot shows fringe amplitude versus delay (vertical axis) and time (horizontal axis) for a compact hot-spot in $3 \mathrm{C}$ source [5]. The WSRT was operating for the first time in
In the 1970s and 8os VLBI observations were still called "Experiments" because any errors in set-up (e.g. feed polarisation orientation or clock failures) would go undetected until correlation took place, usually well after the data was recorded. But by the late 1980s, VLBI had grown into a mature observing mode in Westerbork making use of increasingly sophisticated adding systems and VLBI recording systems, and the $92 \mathrm{~cm}, 18 / 21 \mathrm{~cm}, 6 / 50 \mathrm{~cm}$ and later the MFFEE receiver systems.

During 1977 to 1980 , plans were laid for the establishment of the European VLBI Network (EVN) including a Program Committee to allocate observing time in six sessions each year. The EVN Board was composed of the Directors of the five founding member observatories - WSRT/Dwingeloo, Effelsberg, Jodrell Bank, Onsala, and Bologna. Harry van de Laan represented the NFRA. Ivan Pauliny-Toth (MPIfR) and Richard Schilizzi were the first Chair and Secretary respectively of the EVN PC.

One of the main issues discussed by the Directors in 1980 was the need for a larger data processor than the 3-station MkIII correlator purchased by the MPIfR from Haystack due to come online in 1982. Driving this idea was the requirement for a "real-time" correlator for 8-station geostationary satellite-linked VLBI, the study of which by ESA had been initiated by NFRA in 1977 [6] following successful tests by a Canadian-US collaboration using a Canadian satellite [7]. In parallel with the ESA studies, Arnold van Ardenne, John O'Sullivan and Jan Buiter carried out tests of local oscillator transfer via the Orbital Test Satellite (OTS) in geostationary orbit [8, 9].

Following an exchange of letters between the EVN (spearheaded by Harry van der Laan) and ESA in 1982 on the L-SAT opportunity, the EVN Board decided

CHAPTER 7 VERY LONG BASELINE INTERFEROMETRY: THE EVN, WSRT, AND JIVE 
that L-SAT use would be too expensive and that was the end of satellite-linked VLBI. However, by then, additional existing telescopes had joined the EVN and new telescopes were planned in Italy and Poland, so the requirement for a large data processor remained.

In the ensuing two years, two proposals were developed, the first to upgrade the MkIII processor at MPIfR to 8 stations (estimated cost $10 \mathrm{Mfl}, 4.5 \mathrm{M€}$ ), and the second to develop a new generation (12 station) user-friendly data processor in Dwingeloo (estimated cost $15 \mathrm{Mfl}, 6.8 \mathrm{M€}$ ) [10, 11]. In 1985, the EVN Board decided to create a stronger governance structure and established the European Consortium for VLBI with eight members and a mandate to continue to carry out VLBI observations with the EVN and to seek funding for a large correlator. The nexus surrounding the data processor alternatives was broken in 1984 by a meeting of the NFRA and MPIfR to discuss collaboration on the future large correlator. MPIfR decided that a better match to its mission was to expand its MkIII processor with its own funding, primarily for the scientific use of its staff; this led to the Consortium agreeing in 1985 to seek funding for a new generation processor in Dwingeloo.

In 1986, contact was made with the European Commission in the person of Prof. Paolo Fasella, Director-General of the Science, Research and Development Directorate) by a Dutch delegation consisting Ruurd van Lieshout (ZWO Director), van der Laan, and Schilizzi. Parallel to this, contacts were also made with senior officials in the Dutch Ministry of Education and Science by van der Laan and Wim Brouw. Two years later, a proposal was made to the EC for a 20-station data processor capable of handling observations from all the then possible VLBI stations in the northern hemisphere. The estimated cost was 17.8 M€ (8.1 M€). The EC called in the European Science Foundation for an independent review of the proposal which the ESF Space Science Committee subsequently endorsed in 1989 . This allowed the EC to provide an initial grant from the second Framework Program (FP2) for the European VLBI project in the form of a Penny \& Giles MkIII tape recorder to be installed at the MPIfR data processor in Bonn.

A few months later in 1990, the Dutch government (Education and Science Minister, Wim Deetman) called again on the ESF to establish a review panel on ground-based astronomy to help put the VLBI proposal in context on a European scale. The result was resounding support for the VLBI data processor. Then followed a year-long hiatus in which some exploratory inter-government discussions took place, particularly with the French Science Minister, Herbert Curien. In 1992 the then new Dutch Minister of Education and Science, Jo Ritzen, convinced the EC Vice-President, Prof Filippo Pandolfi, of the desirability of further EC support for the data processor in order to trigger national contributions. This was forthcoming in the form of a $1 \mathrm{M} €$ grant for Human capital and Mobility in FP3.
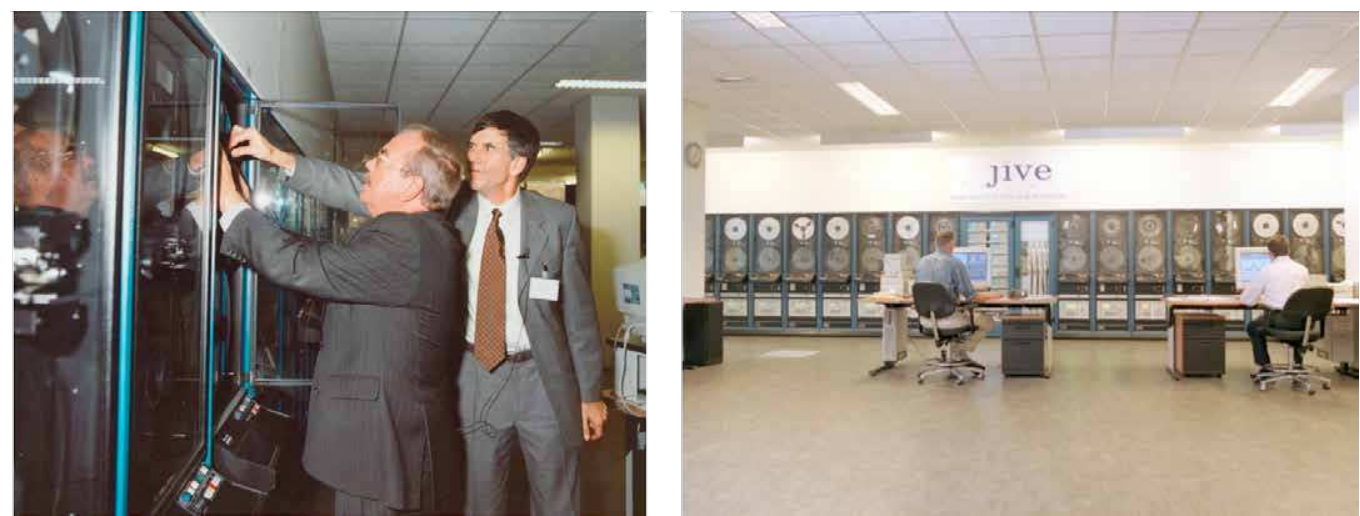

Following this announcement later in 1992, 5.5 M€ was made available by the Dutch Ministry of Education and Science, 0.3 M€ from the French National Centre for Scientific Research (CNRS), and 0.55 M€ from the Swedish Wallenberg Foundation. In addition, the Jodrell Bank Observatory in the UK agreed to provide manpower for the data processor design. And in early 1993, design, prototyping, and construction of 16 station MkIV processor by an international consortium including the Haystack Observatory in the USA began. It ended almost six years later at a cost of $8.7 \mathrm{M} €$ including manpower. Further details of the data processor are given in Section 2.2.

On 22 October 1998, the official opening of EVN Data Processor at JIVE was carried out by "Comissaris van de Koningin in Drenthe", Relus ter Beek (Figure 3).

In 1991, internal discussions in the NFRA led to a decision to propose that the VLBI data processor in Dwingeloo be the focal point of a new European centre for VLBI, which came to be called the Joint Institute for VLBI in Europe (JIVE). At the end of 1993, JIVE was formally established as a Foundation in the Netherlands with Schilizzi as the first director.

1.2 Establishing JIVE as a European Research Infrastructure Consortium (ERIC)

Already in the early days of JIVE, it was a recognized to be desirable to transform JIVE into a truly international entity, away from the Dutch 'stichting' legal entity. JIVE being an outstanding example of a distributed, bottom-up organized European research facility, one can suspect that the EC was well aware of this fundamental problem for JIVE and similar entities. In the $4^{\text {th }}$ Framework Programme, the EC started to support a number of distributed or collaborative facilities like RadioNet in which JIVE and ASTRON featured prominently, and this carried on in the $5^{\text {th }}, 6^{\text {th }}$ and $7^{\text {th }}$ Framework. During which time the need to have light-weight, truly European entities became much clearer. The ERIC construct was established in 2009 and it allows one to register a legal entity in Brussels as a collaboration of member countries.
Figure 3. (left) Commissaris Relus ter Beek, with the help of Jive director Richard Schilizzi, loading a tape on one of the 16 tape drives just before successfully pushing a button to demonstrate fringes from the new data view of the 16 tape drives and auxiliary electronics and data processor operators. 
For JIVE there were many attractions to becoming an ERIC including formal recognition as an excellent European research infrastructure provider, VAT exemption, and a governance structure based on collaboration of countries rather than the joint responsibility of a mixed bag of universities and national research organizations with different objectives. The latter was also the greatest hurdle to the transformation of JIVE to an ERIC; not in all JIVE member countries it was easy —or even possible - to transfer the interest in JIVE to a ministerial level. In a process that took several years, the Dutch representatives at ASTRON, NWO and the ministry made it clear that the transformation into an ERIC was a requirement to continue JIVE operations at the same level in the Netherlands, especially in an era in which the definition and design of the SKA were also consuming resources.

Figure 4. Dutch key players at the JIV-ERIC inauguration on 21 April 2015 From left to right: JIVE director Huib Jan van Langevelde, EC Science director-general Robert-Jan Smits, NWO astronomy domain leader Ronald Stark, ASTRON direcor-general Michael Garrett and Westerbork director Rene Vermeulen.

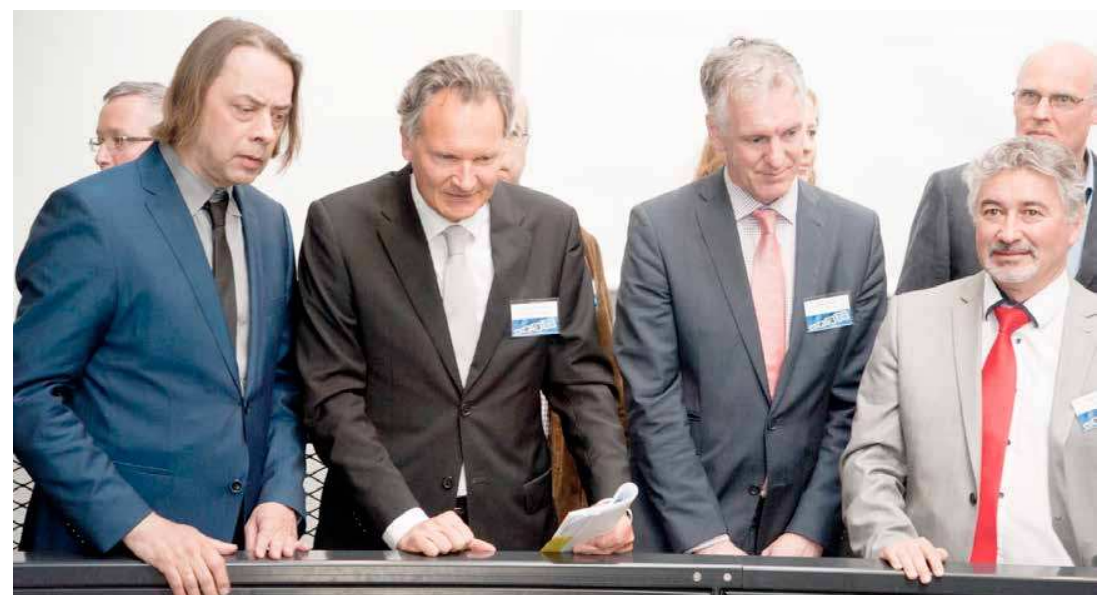

JIVE ERIC was established in December 2014 with four nation members. Since then the membership has increased to six countries and further support comes from research organizations in three more countries, some of which intend to become full members in the future.

1.3 Space VLBI

The early history of space VLBI is described in references [12] and [13]. After the successful participation in the Japanese VSOP-HALCA mission, JIVE and ASTRON staff led by Leonid Gurvits were also eager to contribute to the Russian RadioAstron mission when it was finally launched in 2011. As the EVN decided to accept observing proposals with the Russian telescopes, Westerbork and JIVE staff became involved in supporting various aspects of the mission. The JIVE SFXC correlator was made available on a best efforts basis for correlating RadioAstron observations observed via the normal EVN proposal process. One non-standard project involved procedures developed at JIVE to measure the RadioAstron orbit with simultaneous observations of the spacecraft by a number of telescopes on the ground [14].

\section{VLBI Instrumentation}

2.1 The Tied-Array at Westerbork

Following the first VLBI observations with the Dwingeloo $25 \mathrm{~m}$ telescope in 1976 mentioned in Section 1.1, attention turned to using the large collecting area of the $14 \times 25 \mathrm{~m}$ dishes of the Westerbork array (equivalent to a $93 \mathrm{~m}$ diameter dish) for VLBI. This required additional and VLBI-dedicated instrumentation to be added to the WSRT in order to gain this large increase in sensitivity and involved adding the signals from all 14 telescopes while maintaining phase coherence to create a so-called tied array, which could act as a single element of a VLBI network or array. The term "tied-array" was coined by Wim Brouw in a corridor discussion in 1977 with Arnold van Ardenne and Richard Schilizzi.

The concept is summarised in Figure 5.

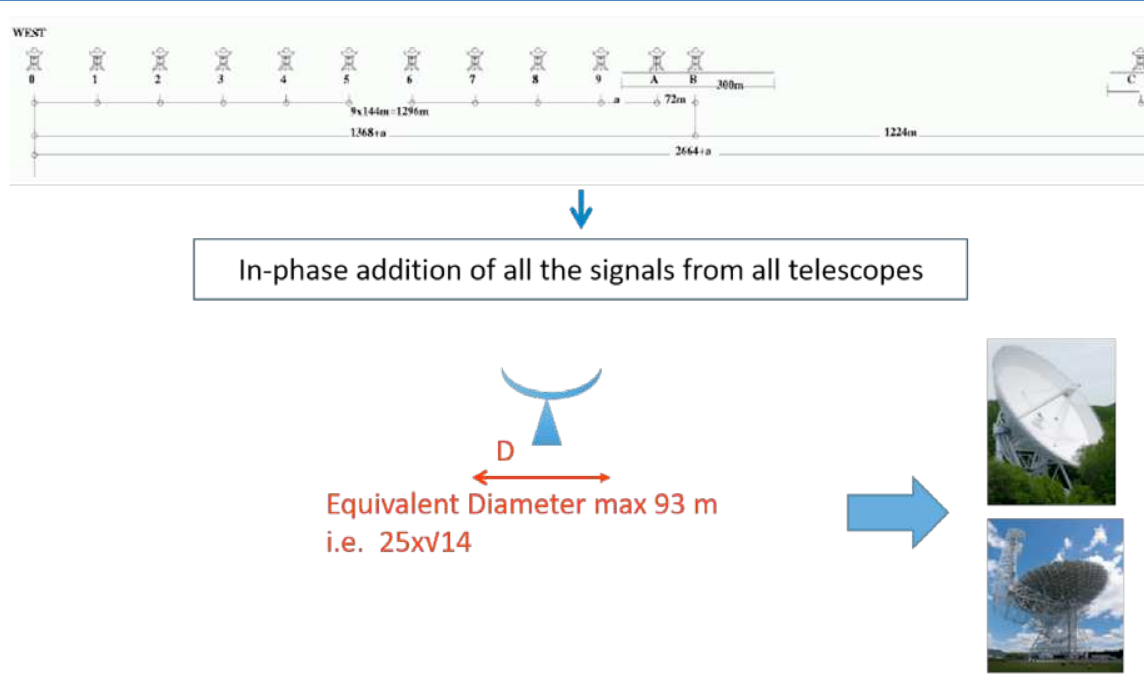

Since this was the first time this had been attempted anywhere in the world, Figure 5. Adding all many questions needed to be answered including i) what loss factors affect the the individual WSRT VLBI coherence for the tied array signal, and what was the resulting effective telescope signals area of the tied array, ii) what were the polarization characteristics of the result - would ideally result ing tied-array and what calibration procedures needed to be developed, and iii) in a telescope with what point would be adopted as the phase centre of the array. Efforts to solve of o3m. This is these problems were carried out in parallel with early tests of prototype units.

This challenging project to implement and test a number of versions of the new tied- array mode lasted from 1978 to 1986. It was led by Arnold van Ardenne and involved a number of SRZM staff including Hans van Someren Greve, Arie Hin, Jaap Bregman, Harm Jan Stiepel, Jan Buiter, Roelof Kiers, Sip Sijtsma, Piet van den Akker, and others. 
Initially, the tied-array system for the $2 \mathrm{MHz}$ bandwidth MkII tape recorder system was implemented on the WSRT DLB. Later versions were implemented first for the DXB, then on the DCB to handle the $56 \mathrm{MHz}$ bandwidth MkIII recorder system, and on the DZB to handle the $112 \mathrm{MHz}$ MkIIIa, MkIV system and e-VLBI (described later in this section). The DZB version was given an official name: the Tied Array Adding Unit ("TADU”). These will now be described briefly.

The tied-array adding units

Four versions of the adding unit were built:

1) 1978: an analogue system [15] built for the Digital Line Backend (DLB) comprising a number of custom units inserted at the point where the telescope signals from the 14 telescopes in 2 polarizations were about to enter the analogue-digital converters of the DLB. These units were made in the Dwingeloo lab mostly by Jan Buiter who remained at the centre of the development of dedicated VLBI equipment for more than three decades. The output of the DLB was digitised before recording on the $2 \mathrm{MHz}$ MkIIc system.

2) 1981: a digital combiner system [16] built to provide output to the MkIIc terminal and which used the DLB for calibration and phase/delay alignment. Understanding how to analyse and calibrate the tied array benefitted from fruitful discussion with Jaap Bregman, then system engineer at the WSRT. This digital combiner anticipated use with the MkIII recorder which had been ordered from Haystack and the digital delay lines then being implemented for the WSRT. However, use of the combiner with the DLB would have limited the input signal bandwidth to the MkIII recorder input to $8 \mathrm{MHz}$ whereas a $56 \mathrm{MHz}$ bandwidth could be recorded. This led to the next step in tied-array development.

3) 1983: the bandwidth bottleneck for MkIII VLBI was removed to a large extent by a digital combiner [17] designed to use the Digital Continuum Backend (DCB) infrastructure and in which the "bulk" fringe rotator was located before input to the correlator. The much more complicated VLBI-specific system used a residual fringe modulator based on the so-called Cordic iteration principle and a subsequent Weaver scheme to recover the folded sideband technique used in the signal conditioning of the DCB. The unfolding inside the DCB was done by digital cross correlation techniques in combination with 90 degrees phase shifting. Photographs of the equipment are shown in Figure 6.

2006: The Tied Array Distribution Unit (TADU) was developed in combination with the DZB as the interface to Marks terminals for VLBI recording and to PUMA II, the Pulsar Backend of the WSRT. It is a flexible all-digital, FPGA-based system that functionally follows the Tied Array Adder Module ("TAAM") in the DZB ADC subsystems [18]. TADU is extremely flexible by design using time variant digital (interpolation) filtering, and allows a variety of output "customers".
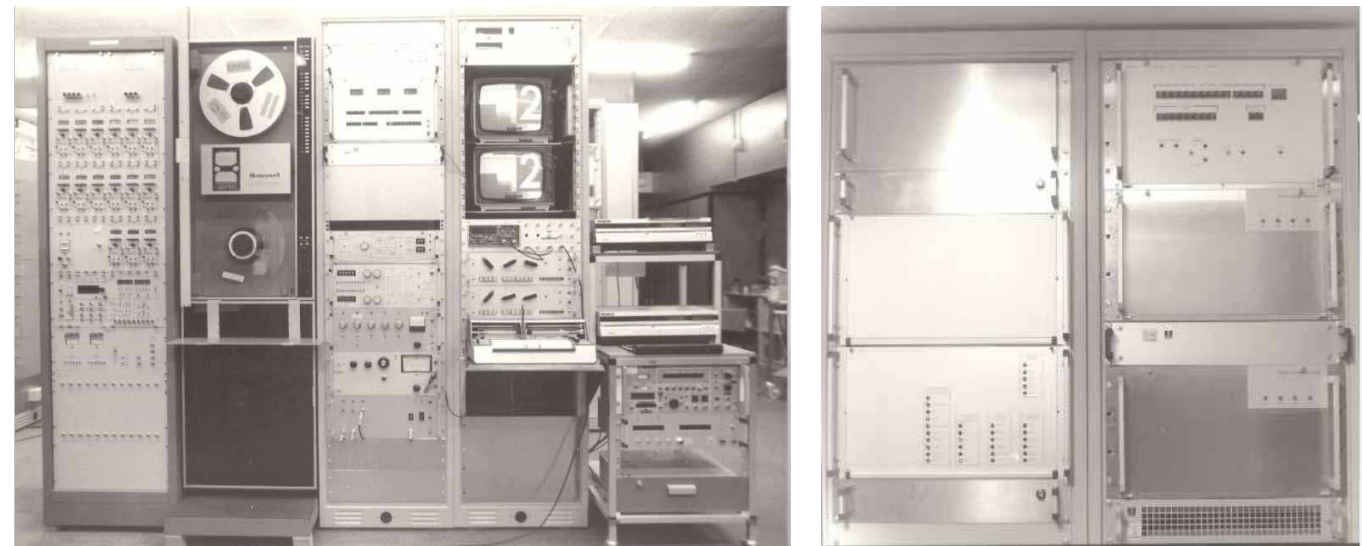

Sensitivity loss in a tied-array

Figure 6. Left: VLB interface equipment - MKIII tape recorder Analogue combiner

The main concern initially [19] was calibrating the level of phase coherence of the added signals at the point of analogue addition knowing that full calibration was only possible using the correlator located "after" the point of addition. In practice, any differential effects proved effectively negligible and others were more important.

One of these factors was the presence of the $6 / 21 \mathrm{~cm}$ cooled receivers in the movable telescopes which were much more sensitive than the receivers in the fixed telescopes (Note: At 50 and $92 \mathrm{~cm}$, all receivers were the same). The cooled receivers dominate the added signal and thereby reduce the effective diameter of the equivalent dish. In practice, the cooled receivers had about $3 x$ lower system temperature, assuming no other losses, so the equivalent diameter ranged between $50 \mathrm{~m}$ (only the cooled receivers on the movable telescopes contribute) and $93 \mathrm{~m}$ (all telescopes contribute with equal system temperature).

\section{Digital combiner}

A more complex issue was the sensitivity loss with the later digital combiners [20]. Extra losses were caused when the digital signals output from the delay system are added and then again resampled for the VLBI recording terminal. For a 1- or 2-bit re-digitized signal from the WSRT as one input to the VLBI correlator, extra losses are introduced that are quantified in reference [20] using probabilistic ("multi-variate”) analysis.

The conclusion is that the most significant degradation losses occur for a small number of telescopes in the WSRT which, say, are 1-bit digitized before addition and re-sampling. In particular, with an even number of telescopes correlated signal power is "lost" in the equivalent "zero" state. It is interesting that only in 2001, over 20 years later, the more general case was solved by Kokkeler et al [21] 
Today, with multi-bit sampling techniques and large numbers of telescopes, this early realization is hardly of practical use!

Location of the tied-array phase centre

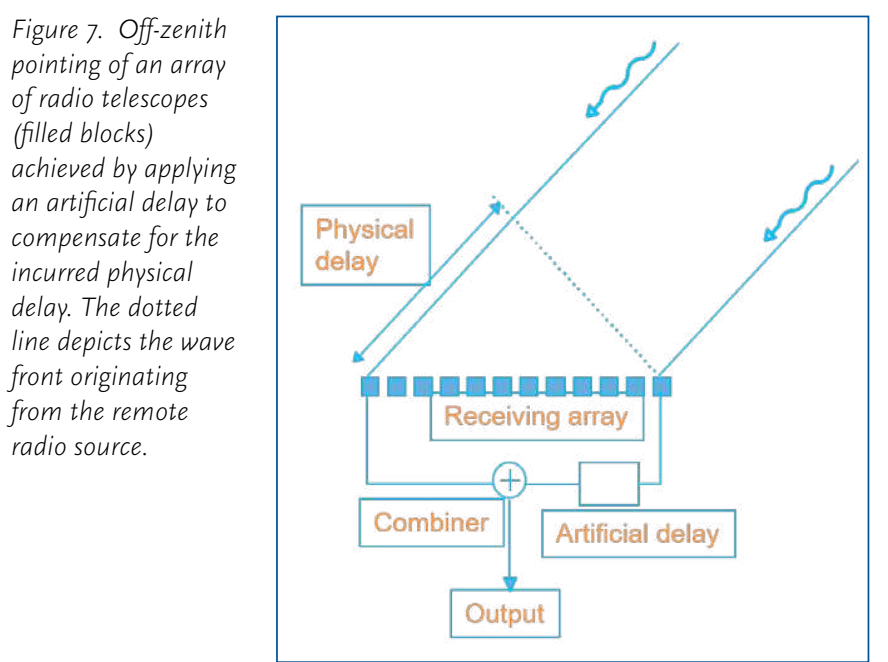

For a single dish telescope, the phase centre is at the focus of the telescope at the point where the reflected power from the telescope is maximum. In an array like Westerbork as in all arrays, the wave front from the remote radio source passes through each telescope's phase centre simultaneously if they all point toward the zenith. Pointing in any other direction is controlled by a delay system that ensures all signals from the same wavefront arrive at the correlator at the same time (see Figure 7 by Felix Smits).

The location of the "common" phase centre of the added array is the physical location of the centre of the array assuming that all receivers have equal system temperature. For much the same reasons mentioned in the discussion of tied-array sensitivity, the apparent phase centre will shift toward the telescopes with cooled receivers along the line connecting all individual phase centres, simply because their "weight" in the added signal is larger. This argument is illustrated in NFRA Note 315 [19] and originates from John O'Sullivan then working at ASTRON. This is not desirable because an antenna reference position is required for the VLBI correlator model and receiver gain or other changes would result in changes in the apparent location of the tied-array phase centre. This in turn would cause changes in length and orientation of baselines to other telescopes in the VLBI array and result in imaging and astrometric errors.

The situation is even more complicated as the WSRT telescope array orientation is East-West and the observed source moves with hour angle in that way i.e. from east to west. In pre-digital days, Hans van Someren Greve, responsible for the online system, implemented the control software such that the proper delay per telescope was achieved by controlled insertion of different cable lengths in the signal paths while observing. By the nature of adding delays, it must be ensured that the delay is always "positive". This was done by defining a "delay-centre" outside the array and in practice at a fixed location to the east of the easternmost telescope. After some analysis, it was possible to show that for VLBI, this centre is also the "location" of the tied array telescope!
When the Philips Computer controlling the analogue cable delays was decommissioned in 1978, an all-digital delay system for the DLB (later the DXB) was introduced. With those digital delays, the telescope signal amplitudes were "normalized" by the converters in the digital system before adding, and gain changes, and phase centre changes became largely irrelevant

A final note: the massive computing power of LOFAR allows formation of multiple tied-array beams for pulsar work. This was foreshadowed by the inclusion of an "auxiliary" output for the first digital combiner [16] which was used for the first pulsar observations with the WSRT (see Chapter 5 on the "Time varying Universe").

\subsection{The EVN MkIV correlator at JIVE}

The development of the EVN MkIV correlator required the bundling of a range of expertise [22]. The correlator design was in the hands of Albert Bos (ASTRON), Sergei Pogrebenko (JIVE), Alan Whitney (MIT-Haystack) and Bryan Anderson (Jodrell Bank), and was eventually used in four data processors, a JIVE (EVN MkIV correlator), ASTRON (DZB correlator), Haystack (Haystack MkIV correlator) and Bonn (MPIfR MkIV correlator). A new chip was designed and manufactured, and local ASTRON expertise was employed for the configuration and data routing on the correlator boards. A UK company, Penny \& Giles Pty Ltd supplied the tape drives and the Station Units under the leadership of Steve Parsley who later joined JIVE. Moreover, it was decided that the EVN correlator was going to run on totally new software, its design being led by Roger Noble from Jodrell Bank. With the emphasis for the EVN correlator on astronomical applications, the output data format was chosen to be the AIPS++ MeasurementSet definition.

The introduction of the MkIV correlator not only provided for more sensitive observations with increased bandwidth, but the use of detailed CALC/SOLVE geodetic models provided accurate interferometer models in the direction of both target and nearby calibrator. This enabled phase referencing between the two and allowed the detection of weak targets, as well as providing very accurate positions for astrometric applications.

After its opening in 1998, the EVN MkIV correlator at JIVE rapidly became the operational heart of the European VLBI Network. By 2000 , essentially all EVN observations and $\sim 5 \% \%$ of global observations were correlated at JIVE. A professional user support structure had been put in place in Dwingeloo as well, funded for a substantial fraction by the RadioNet TNA (transnational access) funds, a mechanism though which the EC basically bought a fraction of the observing time for users from all over Europe. It allowed JIVE to implement a rigorous data scrutiny practice and support users in all stages from proposal preparation to data processing. Users were also encouraged to come to Dwingeloo, stay in the guest house, and work with the support scientists on their data. 


\section{Closing the holes}

Not always is an apparent good idea translated into hopeful dreams and subsequent realities. In this case, it started with the demand for higher frequency VLBI, but VLBI wasn't the only motivation for improving he reflector mesh. Improvements to performance of the feeds and receivers was hindered by the inherent loss of the reflector mesh. The loss of reflectivity with increasing

frequency has a negative impact on

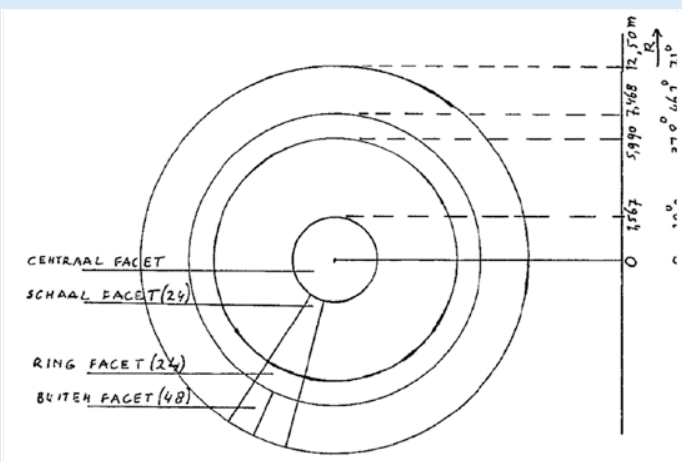

the antenna coupling efficiency, and also, for the same reason, the loss of reflectivity is accompanied by an increse in $u$ "of the "hot" environment. As the ratio of these ratio of these two are important for por overall sensitivity, it was important to look into possibilities for mprovement.

This was done by exploring two routes with the first to look into painting the mesh with conductive paint This would partally fill the pain. This would partially fil the holes and therefore reduce the transmission. After lots of experimenting with different paints and performing microwave measurements as a function of angle of reflection in the labs (lots of young- er people still wonder about the purpose of the waveguide segments with varying widths), it was conclud. ed that about $40 \%$ reduction would result at $5 \mathrm{CHz}$, and less at higher frequencies.

The second approach was to close the mesh by gluing thin stainless steel slabs on the panels! Questions were of course: Which panels? Some or all of them? This was studied by looking at the mechanical layout of the panels (see Figure 1 taken from the 1978 "Internal the panels from the centre ("apex") out to a $6 \mathrm{~m}$ radius comprising $25 \%$ of the physical area, a $60 \%$ reduced this improved ing the $75 \%$ of the remaining area.

The obvious other question was regarding which glue should be used and could it withstand the Dutch climate? This was especially on our minds in view or our experience with the loosening of the mesh on their frames (see insert by Wout Beeramp). So outside (n) the lab, spare panels with stainless steel slabs glued with the selected glue, were placed and tested in all conditions including intense solar radiation etc. Until very recently these panels were still outside near to the Dwingeloo telescope and people were wondering again what they were doing out there! Of course, they could not know that the idea was not pursued because with all the slabs plus paint on the WSRT antennas the additional windload turned out to be unacceptable. Note"). It turned out that by closing
In this way, JIVE built up a reputation as the 'friendly, local correlator', where the staff would help to get the most out of the EVN data. In this way its support staff also had an opportunity to become part of the scientific teams around the EVN and many of the JIVE postdocs were able to progress their career in science after their JIVE post. In order to support astronomical use, much attention was paid to improving the calibration of the EVN and the support staff also monitored network performance through a reliability index. With external funding the PC board-based Integrator ("PCInt") was developed to overcome the limits on bandwidth and time sampling, research was done on ionospheric calibration, and JIVE staff developed ParselTongue to allow users to script large or innovative data reduction problems [23].

\section{$2.3 \quad e-V L B I$ and parallel developments}

After the important introduction of phase referencing in the nineties [24], an other revolution took place in the EVN around the turn of the century. Probably this was a silent revolution to most astronomers, as the sensitivity, and thus the advertised scientific potential of VLBI did not change dramatically at first. However, very noticeable to the users (and operators) were the improvements in robustness, calibration and flexibility when VLBI operations moved away from spinning tape as the recording medium. The transition to disk units rather than tapes was the first and maybe the most noticeable innovation that used off-the-shelf components, but not the last. The introduction of fibre-linked e-VLBI, software correlators and high-speed digitizers would all open up capabilities for new research areas. The WSRT often played a special role in these developments because of its close location to the correlator at JIVE, and its unique nature as the only linked element interferometer in the EVN, capable of providing simultaneous images.

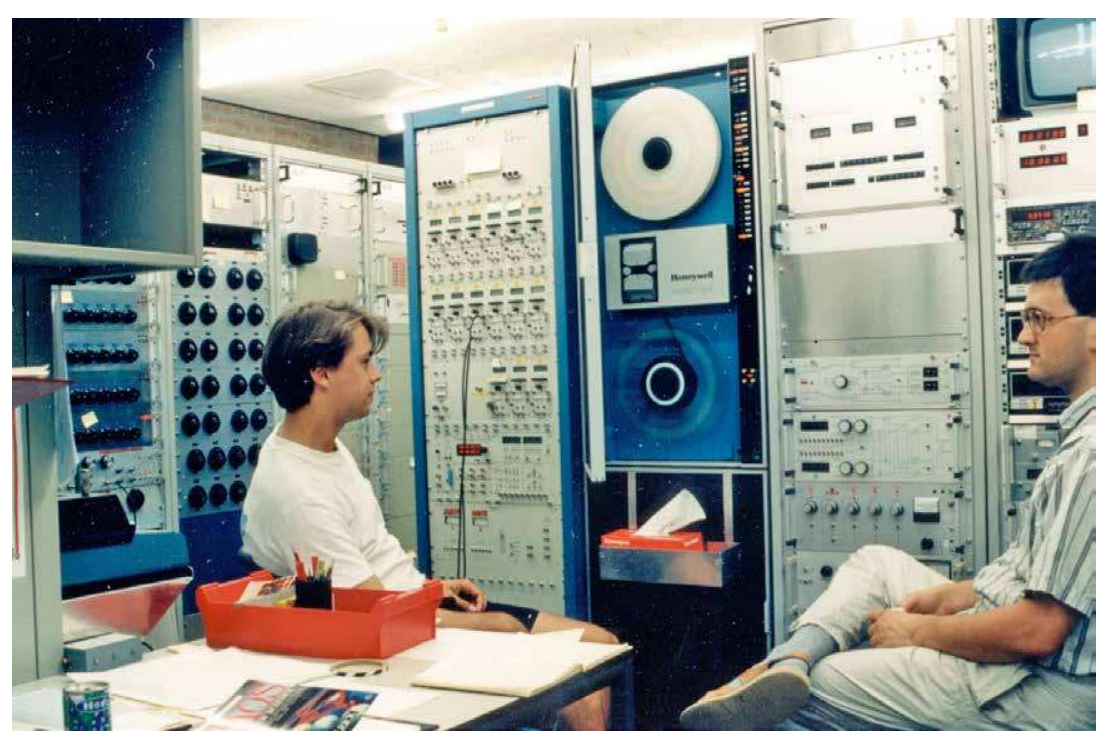

Figure 8. For the global campaign on $\mathrm{SS}_{433}$ in 1987 students spent the night at Westerbork to help changing tapes. Huib van Langevelde and Anton Jongeneelen doing their duty in the late eighties. 
In 2001 MIT-Haystack introduced the MkV data acquisition and playback units, which were no longer using spinning tapes for recording. Instead a PC board was used with a special card that distributed the data in parallel over eight hard disks mounted in a special chassis. These units were connected to the telescope outputs, as well as to the input of the correlator. The motivations for $\mathrm{MkV}_{5}$ were cost savings on the recording medium (disk-packs vs tape) and transport costs, no requirement for the almost annual replacement of expensive write-read heads on the tapre machines at the stations and correlators, and reliability. JIVE and WSRT staff worked together to update the telescope and correlator interfaces to accommodate MkV.

The introduction of disk recording did magic to the operations of the VLBI network. Immediately the data error rates were much better and no longer required fine-tuning of tape alignments and (reading/writing) head calibrations. Moreover, the recording efficiency improved, even if the disks systems did not readily offer the same bandwidth, because the disk units were immediately ready for recording and required no tape conditioning. At the correlator, data read-back became direct access, where before complex tape priming was required to align all station recordings at the same micro-second of the original observation.

In addition, with the data stored on 'normal' hard disks, the data had become directly accessible for the station or correlator staff. It made it possible to implement a number of diagnostics to study the statistics of the digitizers and the telescope passbands. It also made it much easier to send short data samples to a correlator and do the ultimate check of VLBI by making sure that fringes were achieved. Looking ahead to the internet era, JIVE staff already made a first attempt to implement a software correlator for fringe verification around the turn of the century. The so-called ftp-fringe tests introduced in 2004, often using the WSRT as the "home station" but the bottleneck for such tests was the very restricted internet bandwidths for some stations, based on telephone lines.

On the initiative of Richard Schilizzi, discussions started in European forums in 2000 on the possibility of implementing e-VLBI using the broad-band, fibre-based infrastructure then under development for European science in order to connect the telescopes to the correlator. With the help of SURFNet a pilot project was carried out with a limited number of stations. Then, JIVE Director Mike Garrett led an EC proposal named EXPReS (Express Production Real-Time e-VLBI Service, funded in 2006) to turn the full EVN into a real-time VLBI instrument that could track transient phenomena in the Universe. It promised to deliver e-VLBI functionality for European astronomers who would be able to inspect the state of their (transient) targets within hours of the observations. That new functionality did require major adjustments to the correlator workings; this was a machine that was designed to run on a rigid reconstructed observing time and the correlator processes were designed to ask for the data from specific times. But for e-VLBI it also needed to correlate data with time stamps streaming into Dwingeloo in real time. Obviously, the project also addressed the connectivity and bandwidth issues. For this aspect, the Dutch SURFNet was an important partner; they were instrumental in helping to establish the connections to Dwingeloo and Westerbork, but moreover they had an ambition to make the Netherlands an important hub in international networking. When the e-EVN switched on in 2005, the Internet traffic at the Amsterdam Internet exchange doubled.

The EC project could not pay for the operational investments needed for the fibre connections from the EVN telescopes to the broad-band network, which was often more than a "last one mile" away. However, gradually the majority of the telescopes came on-line with bandwidths increasing from $128 \mathrm{Mbps}$ to Gbps. Being close to JIVE, the Westerbork telescope had a special place; it was linked on a dedicated fibre connection, providing a perfect testbed for pioneer ing protocols for the data relays. Many tests were done with different routing hardware, internet protocols and clever data packaging [25]

The EVN also adopted new operational modes to engage in e-VLBI. Dedicated e-VLBI observing days accommodated tests and user experiments. At first, the users had to come forward with compelling arguments why they needed quick access to their data, later - when the bandwidth was competitive to recorded VLBI - any experiment could be transferred to the e-VLBI days. Some examples of the resulting science are listed in Section 3.

Another activity funded in the EXPReS project was the development of a distributed correlator platform, based on high-performance computing nodes, in which Westerbork staff collaborated. Arguably, the data transport requirements could be distributed differently from the traditional all-telescopes-to-one-correlator star configuration. Research was done on how data copies could stream from each telescope to each other in a network configuration. The load-balancing, distribution software that was developed never became operational, but the linux-based, software correlator, derived from the Huygens processing algorithm (see Section 3) later became the SFXC platform [26].

After EXPReS, the EC also granted funding to NEXPReS (Novel EXplorations Pushing Robust e-VLBI Services) in 2010, a project led by JIVE Director Huib Jan van Langevelde, for which the major deliverable was to ensure the European collaborations that had started under GEANT would be sustained. It allowed JIVE and the EVN to continue support for e-VLBI at competitive bandwidths. The R\&D shifted towards defining a recording standard independent of MIT-Haystack, who were already announcing MkVI data acquisition units. The focus was on using standard Linux data file systems on these units, such that more flexibility was possible between recording and streaming data. At this time JIVE also developed new data acquisition platform software to allow robust integration of recorded and streamed VLBI modes. Again, the WSRT proved to be an important testbed. 
In early 2013 the MkIV correlator at JIVE was switched off and dismantled after almost 15 years of operation, its functionality replaced in the previous years by a Linux cluster running the SFXC software. JIVE and ASTRON staff, led by Arpad Szomoru and financed by EC project RadioNet, also developed an FPGA correlator named the UniBoard platform [27]. The UniBoard was later used for LOFAR station processing, the Apertif system at Westerbork and considered for processing SKA data. The SFXC correlator, completely based on locally developed software, proved to be a huge success for JIVE and the EVN. It allowed new science modes for wide-field imaging, time domain processing and spacecraft applications (see Section 3). Moreover, it proved vitally important that JIVE staff could easily control the channelization of input data streams, as cheap and powerful digitization technology led to a proliferation of telescope data acquisition systems.

\section{Time Adventures}

Lout Sondaar, Albert Jan Boonstra, Arnold van Ardenne

In radio interferometry the combined receivers act as a phase-coherent system. In practice this is done by locking all analogue and digital receiver electronics to the same frequency reference source. For local observations using only the WSRT telescopes, the requirements for such a reference source are differential and modest. How ever, for VLBI and pulsar timing observations, a very accurate atomic clock is needed to ensure coherency with radio telescopes throughout the world.

In the early eighties, the Westerbork VLBI system was in dire need of its own precision clock. Until then precision time was delivered through a rather complicated method involving synchronization through television with help of the Netherlands Measurement Institute ("VSL"). The budget to acquire a clock, a state-of-the-art Hydrogen maser, was beyond our means. A more elaborate scheme came to mind which involved close collaboration with the Swiss Clock Research Laboratories in conjunction with their company Oscilloquarz in Neuchatel. They had an early prototype of a working maser which was available to be modified for use in Westerbork for a fifth of the price. This was win-win for all as in principle it had already served its research purpose

Therefore Lout Sondaar, working at to Neuchatel in 1983 to adapt the maser for use in Westerbork. The picture shows Lout standing beside the complete maser in the Neuchatel Labs.

The principle of the maser was to in the so-called physics package As this is an atomic transition, its frequency is ultra-stable and has ideally suited as a precision clock. This $21 \mathrm{~cm}$ line corresponds to the our Technical Laboratories, moved generate the $21 \mathrm{~cm}$ line frequency (the large structure in the picture).

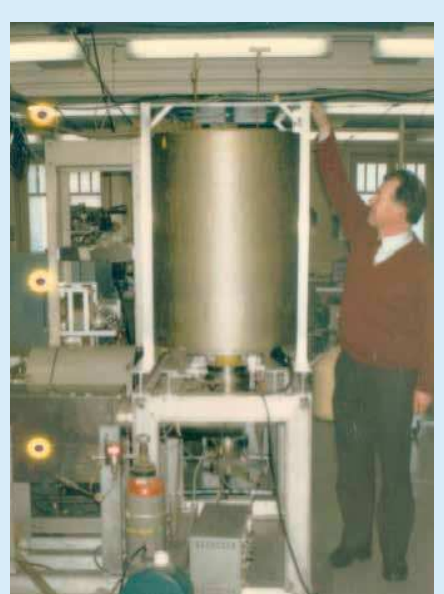

Lout Sondaar preparing the first Westerbork Hydrogen Maser in the Oscilloquarz Labs in Neuchatel early 1984.

one we are "commonly" measuring using the WSRT and our own receivers and hence improvements were made to $21 \mathrm{~cm}$ receiver of the clock by our engineers.

In March 1984 the LSHG-H passive $\mathrm{H}$-maser was shipped to Westerbork assisted by Harm Jan Stiepel, the electronics expert in the Observatory. Weighing close to $1000 \mathrm{~kg}$ the maser package allowed for only a few $\mathrm{cm}$ tolerance of the suspension springs of the available truck making it a truly delicate ride to the Netherlands.
Continuing the feat of fine engineering, the maser was successfully installed in the temperature stabilized basement area of the Obsevatory by April 1984. It afterwards performed well during 15 years of continuous operation!

With the nineties-upgrade of the WSRT system, including the extension of the frequency range to the $3.6 \mathrm{~cm}$ geodetic VLBI band, an up grade of the central frequency and time reference was needed. This became even more urgent as the reconditioned at that point serv as WSRT hydrogen maser, was beginning to show its age! A comparison of hydrogen masers on the market led to an attractive 'turnkey' solution. In 1999 the DATUM (currently Microsemi) MHM2010 maser was installed at the same basement. After switching the machine on, only a two-week stabiization period was needed before the maser could be used as the new maser clock showing a solid frequency stability of $2 \times 10^{-15} \mathrm{in} \mathrm{a}$ 1000 second interval.

This long period of delivering stable and precise time, and the collab-

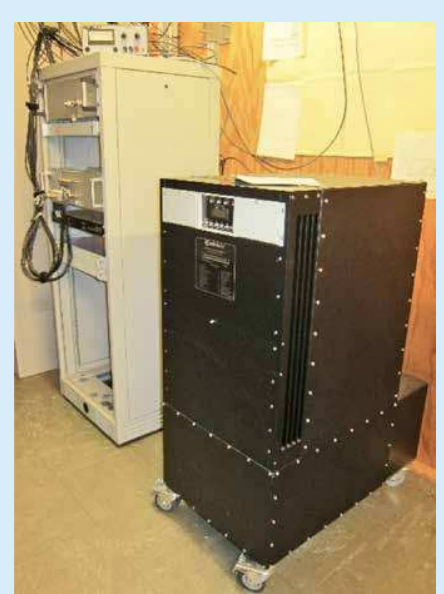

The Datum H-maser in the temperature stabilized room in the basement of the Westerbork Observatory building now in use for almost zoyears! Note that further maser evolutions led to much smaller ones used in the Galileo navigation satellites.

oration with the geodesy group of the Technical University in Delft, resulted in the Observatory "time" and precise location becoming part of the International CNSS Service Network in 1998.

Reference:

The Hydrogen Maser LSHR $\mathrm{H}_{3}$ in Operational use at the Westerbork Synthesis Radio Telescope, L.H. Sondaar, First European Time and Soquency Forum ncon, Fr., 1987 


\section{Science}

By its nature, very high angular resolution VLBI requires high brightness targets (high intensity radio emission from a small solid angle on the sky). Therefore, astrophysical sources that emit through non-thermal emission processes are the prime targets for mas-scale studies. Before phase referencing techniques were established, the additional requirement was that sources needed to be detected within the coherence time, typically 2 to 10 minutes. So traditionally, bright Active Galactic Nuclei (AGN) and their jets have been a prime target for VLBI.

Zooming in on AGNs, VLBI observations were key in establishing that relativistic jets were launched from close to the central massive Black Hole. Studies focused on studying jet physics, beaming ratios and superluminal motions. Important progress in the EVN was obtained in the field of Gigahertz Peaked Spectrum sources and Compact Symmetric Objects (e.g. Figure 10). There was a joint interest from JIVE, ASTRON and staff at the Dutch universities to disentangle the orientation and evolution of active radio sources.

Another area of joint expertise was the study of (HI) absorption associated with the gas surrounding the AGN. In fact, the first science result with the new JIVE MkIV correlator revealed the $\mathrm{HI}$ in $\mathrm{NG}_{42} \mathrm{G}_{1}$, on scales that had just become accessible in the optical through observations by the Hubble telescope [28]. In synergy with the Westerbork telescope, that could access a wide range of redshifts, many galaxies were targeted for HI absorption VLBI.

Also sparked by the observations with the Hubble telescope, were radio observations of its deep fields. Long integrations in the optical and radio showed a cosmic zoo of galaxies, some of them radiating from the early days of the universe. After Westerbork, VLA or MERLIN surveys, VLBI observations were called for, in order to understand the nature of their radio emission. But time and bandwidth averaging would limit the field of view to a single galaxy target, while in principle the primary beams of the individual telescopes encompass many sources [29]. With target lists from the low-resolution surveys in hand, clever correlator modes at the JIVE correlator were introduced to facilitate such wide-field studies.

The early motivation to study the radio emission in galactic compact objects was that this was an opportunity to study jet physics on much shorter timescales. A famous campaign was the monitoring of SS433 in 1987 with VLBI $[30,31]$. Over the years the interest shifted from studying the jet physics and launching mechanisms to making out the stellar evolution that led to the various exotic objects. As is the case for pulsar origins, VLBI makes important contributions to this research by measuring parallaxes and proper motions.

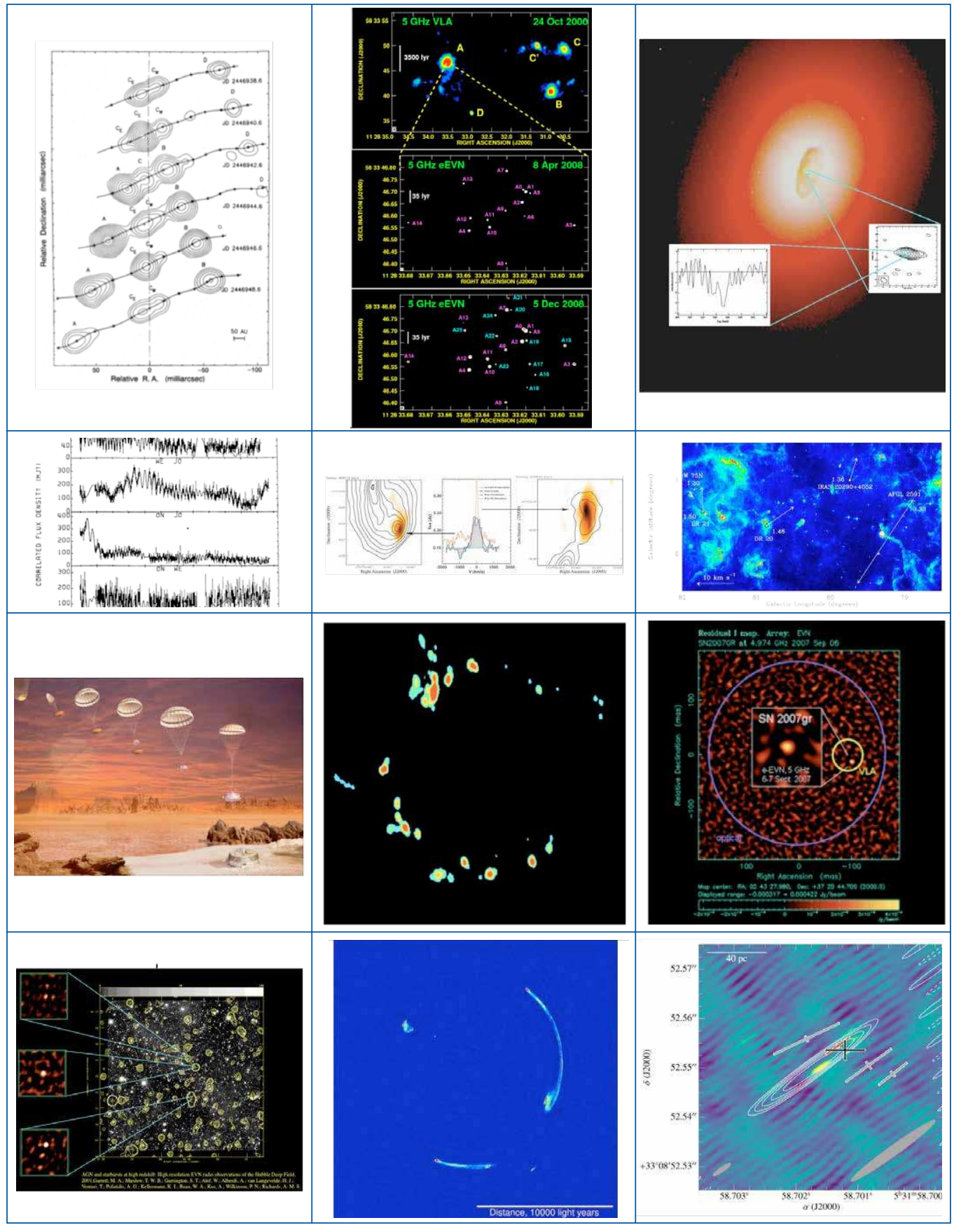

Figure 10. left to right: Some of the iconic images of the EVN: SS433 from [30), supernovae in IC694 [35], HI absorption in NCC4261 [28], the $\mathrm{CSO}_{3} \mathrm{C}_{343.1}$ [36], HI absorption in 4C12.50 [37], distances and proper motions of methanol masers in the Cygnus region [33], an artist Impression of the Hupgens lander [38], a ring-shaped methanol maser [39], the VLBI detection of SN2007gr [34], VLBl sources in the HDF-N [29], global VLBl image of a gravitational lens [41], the localization of the repeating FRB [42]. See the text for explanations of the acronyms. 
High brightness emission from molecular masers that can spontaneously exist in the low-density interstellar medium, make for another category of interesting VLBI targets. These masers are signposts for regions where infrared emission excites molecules faster than collisions with other molecules relaxes them, such as circumstellar regions around evolved stars or star forming regions, or even the dense parts of active/starburst galaxies. The EVN is particularly sensitive to $\mathrm{OH}$ emission from starburst galaxies and IR stars, even though the $18 \mathrm{~cm}$ part of the spectrum has been compromised in many places by manmade interference.

Additionally, the EVN excelled for many years in observations of the $6.7 \mathrm{GHz}$ methanol maser. This ubiquitous maser was only discovered in 1991 and not many telescopes had receivers that covered this transition. In fact, coverage of the line was also not realized in the MFFE receivers of the WSRT, but one dish could be outfitted. And so, together with the EVN, a major contribution was made to studying this transition which was uniquely associated with the earliest stage of high mass star formation. The EVN demonstrated that the masers were linked to the accretion and outflow mechanism on scales of something like a thousand astronomical units, sometimes showing ring-like configurations [32]. With the phase referencing technique, it proved even possible to measure parallax distances to some of these sources [33]. And EVN users showed how high spectral resolution could be used to prove that magnetic fields are important in controlling the formation of high mass stars [34].

The introduction of e-VLBI raised interest in studying transient phenomena, which has turned out to be a very exciting subject. Initially the targets were galactic black holes or other compact objects, particularly those with binary companions. But extragalactic targets were also showing transient phenomena accessible to VLBI. It turned out that the extended radio emission in some nearby starburst galaxies could be resolved into the radio shells of individual supernova by VLBI [35] Invisible in the optical, VLBI could be used to obtain a census of the high mass stars in colliding galaxies.

Following the alerts from robotic telescopes monitoring Gamma Ray Bursts, the EVN started to target these objects. Pinpointing their host galaxies was one objective, but VLBI can in principle also distinguish various explosion models by resolving the source, either directly or through the scintillation properties, as a function of days since the explosion [40]. In these studies the inclusion of the Westerbork array was often crucial; not only did it provide fantastic sensitivity, but also crucial simultaneous lower resolution data, important for calibration and interpretation of the VLBI results.

A triumph of the expertise that was built up in Dwingeloo on correlators, was the VLBI pinpointing of the repeating Fast Radio Burst in 2017 [42]. The FRB phenomena was discovered about a decade ago when the Parkes telescope discovered single millisecond bursts from unknown objects. When Arecibo con- firmed that one such source repeated, a world-wide campaign started to pinpoint the source of these radio flashes. The VLA associated the repeater with a dwarf galaxy at cosmological distances, but the EVN could even associate it with a specific nebulosity in this galaxy as observed with Hubble. It required very complex data processing at the SFXC platform, which was outfitted with the capability to gate very short visibilities after correcting the signal to compensate for the record-high dispersion between the source and the telescopes.

The option to implement such special functionality had been inherent to the SFXC platform from the start, when developed for space applications. Indeed, man-made transmitters on board of spacecraft are also bright enough to do VLBI, even if their detection requires the largest telescopes in the world. The most outstanding achievement in this field remains the VLBI tracking of the Huygens lander as it descended in the atmosphere of the Saturn moon Titan on 14 January 2005 . The ultimate objective was to measure the trajectory of the probe as its motion was impacted by the winds in the atmosphere of this moon that may resemble planet Earth in original composition. Indeed, led by Leonid Gurvits, JIVE scientists published a detailed trajectory later after detailed analysis of the data [43]. However, the biggest visibility for the team came about few hours after the descent, as only real-time detection by the VLBI telescopes could confirm the proper working and time of touch down of the Huygens lander.

\section{The future}

With the advances in digital devices continuing to offer upgrade options, it is clear the VLBI field will deliver more discoveries in the future. And as SKA (and its precursors and pathfinders, like LOFAR) are engaging more astronomers in radio science, there are initiatives in many countries to establish a radio astronomy station. One can expect future VLBI telescopes to deliver enormous instantaneous bandwidth as both direct digitization and wide band receivers are being developed (BRAND EVN, a research activity in RadioNet). More processing and storage capacity will allow the VLBI correlators to offer increasing flexibility for the user products, for example time domain products and multiple, large fields of view or high spectral resolution.

One major cost item for establishing new telescopes is the need to have not only data acquisition instruments at the telescope, but also a highly precise maser clock for time and frequency stability. A hydrogen maser atomic standard is a fundamental requirement to ensure that only atmospheric fluctuations set the calibration intervals, a telescope with poorer stability is useless for phase referencing observations. A pilot project funded by the EC in the ASTERIC (Astronomy ESFRI and Research Infrastructure Cluster) project, is underway to test the transfer of precise clock information between two telescopes over the fibre network. The aim is to do a test transferring the clock signal from the maser at the Westerbork telescope to the Dwingeloo telescope, bringing it back 
as a VLBI element after some 40 years! This same research is informing the design of the signal and data transport sub-system of the SKA.

But of course, the main cost item, certainly in modern times, for constructing a new VLBI telescope is the steel construction. Fortunately, space exploitation also requires large dishes and some of the expansion of the EVN (particularly in China, but also partly for the Sardinia telescope) has been motivated this way. Moreover, in other places, dishes previously used for other (communication) purposes are being retrofitted for VLBI, in Latvia, Ghana and possibly the Portuguese Azores. The case for retrofitting retired communication antennas in African countries is particularly compelling. Such an African VLBI Network complements the SKA_1 with long baselines, providing the capability to pinpoint transient phenomena, measure accurate pulsar and maser positions and distinguish AGN from starburst galaxies. But it is also a chance to involve African scientists and engineers in the inspiring and innovative trade of radio astronomy.

With the Apertif upgrade [See Chapter 15 of this book], the parameters of the WSRT element in the EVN have changed. The phased array is now only available at L-band, albeit with a larger bandwidth than before. For all other bands, MFFE and $5 \mathrm{~cm}$, only a single telescope can participate in the EVN. But the Apertif system, certainly the transient trigger functionality, has important synergies with VLBI and it even has been considered to connect each individual WSRT dish to JIVE for transient and wide field imaging. Maybe one day even VLBI will profit from the multi-beam capabilities on the WSRT. It is hard to predict what the fate will be of the Westerbork array by the time the EVN turns 50 in 2030 , but with the continued interest in global VLBI, it seems reasonable to argue that the historic investments in the sturdy steel Westerbork dishes will still be serving science.

\section{References}

1] R. Porcas, "A history of the EVN", 2010, in "10th European VLBI Network Symposium and EVN Users Meeting: VLBl and the new generation of radio arrays", PoS(10th EVN Symposium) o 1

2] R. S. Booth, "The origins of the EVN and JIVE: Early VLBI in Europe", 2013, in "Resolving the Sky Radio Interferometry: Past, Present and Future" (M.A. Garrett and J. C. Greenwood eds), p.5I

[3] R. T. Schilizzi, G. K. Miley, A van Ardenne et al., "High resolution observations of the compact central component in the giant radio source 3 C 236", 1979, A\&A 77

[4] R. T.Schizzi, "A repon on ViBou in Europe", 1977, NFRA Int. Techn. Rep. No 147

F] V. Kapahi, R. T. Schilizzi, "VLBI observations of hot spots in the lobes of distant radio sources", 1979, Nature 277610

"Very Long Baseline Radio Interferometry Using a Ceostationary Satellite", 1978, .

[7] J. L. Yen, K.I. Kellermann, B. Rayhrer et al. "Real-Time Verr-Long-Baseline Interferometry Based on the Use of a Communications Satellite", 1977 , Science 198289

8] A. van Ardenne, J.D. O'Sullivan, "VLBI Clock/Phase comparison using a Ceostationary Satellite", 1982, Proc. VLBI Techniques, pp 459-467, Toulouse, ISBN 2-85428-087-3

9]A. van Ardenne, J. D. OSullivan, A. de Dianous, A High-Precision Phase-Comparison Experiment Using a Geostationary Satellite", 1983, IEEE Trans. Instrum. and Meaurement, Vol IM-32, Nr.2, pp $370-376$
[10] R.T. Schilizzi, A. van Ardenne, A. Bos, J.D. Bregman, R.H. Harten and H.W. van Someren Greve, "On the Use of Westerbork Facilities for a VLBI Network Centre", 1982, NFRA Int. Techn. Rep. no. 165, European VLBI Processor Memorandum

[11] R.T. Schilizzi, "Proposal for a European VLBI Network Centre in the Netherlands", 1983, Europea VLBI Processor Memorandum 2, 1983.

[12] R. T. Schilizzi and L. I. Gurvits, "The Westerbork Telescope and Very Long baseline Interferometry", 1996, in "The Westerbork Observatory, Continuing Adventure in Radio Astronomy" (E. Raimond and R, Genee (eds), p. 127

[13] R. T. Schilizzi, "A short history of space VLBI", 2013, in "Resolving the Sky - Radio Interferometry. Past, Present and Future" (M. A. Garrett and J. C. Greemwood (eds), p. 99

[15] A. van Ardenne "Proposal for the realisation of Westerbork as a Tied-Array for small bandwidth VLBI Tape Recorder Experiments", 1978, NFRA Note 262

16] A. van Ardenne, "Description of the Digital Combiner", 1981, NFRA Note 362

17] A. van Ardenne, "Proposal for the realisation of a broadband telescope-MkIII Terminal Interface", 1981, NFRA Note 363 (in Dutch)

[18] ]. Dromer, A.W. Cunst, "Technical TADU-max final report", 2006, ASTRON-RP-076

[19] A. van Ardenne, "Calibrating the Tied Array in the Case of Analog Summation of Analog Signals", 980, NFRA Note 315

20] A. van Ardenne, "Sensitivity loss with Westerbork as digital tied array. A first assessment", 1979,

[21] A. B. J. Kokkeler, P. Fridman, A. van Ardenne, "Degradation due to quantization noise in radio astronomy phased arrays", 2001, Exp. Astron, "1133

22] R. T. Schilizzi, W. Aldrich, B. Anderson et al., "The EVN-MarkIV VLBI data processor", 2001, Exp. Astron. 1249

23] M. Kettenis, H. J. van Langevelde, C. Reynolds, and B. Cotton, "ParselTongue: AIPS Talking PYthon", 2006, in "ADASS XV", ASPC 351497

24] A. J. Beasley and I. E. Conway, "VLBI Phase-referencing", 1995, in "Very Long Baseline Interferometry and the VLBA", ASPC 82327

25] P. Boven, "e-VLBI Networking Tricks", 2009, in "Science and Technology of Long Baseline Real-Time Interferometry: The 8th International e-VLBI Workshop", PoS (EXPReSOg) 36

[26] A. Keimpema, M. M. Kettenis, S. V. Pogrebenko et al., "The SFXC software correlator for very long

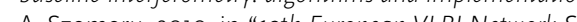

A. Szomoru, 2010, "in: ath European VLBI Network Symposium and EVN

28] H. I. van Langevelde, Y. M. Pihlström, I. E. Conway et al., "A thin HI circumnuclear disk in NGC 4267", 2000, A\&A 354 L45
M. A. Garrett. T. W. B. Muxlow, S. T. Garrington et al., "ACN and starbursts at high redshift: High resolution EVN radio observations of the Hubble Deep Field", 2001, A\&A 366 Ls

Bo] R. C. Vermeulen, R. T. Schilizzi, R. E. Spencer et al., "A Series of VLBI Images of SS433 during the

Outbursts in May/June 1987", 1993, A\&A 270 "177
[31] R. C. Vermeulen, V. Icke, R. T. Schilizzi et al., "Evolving radio structure of the binary star $S 5433$ at a resolution of 15 marcs", 1987, Nature 328309

[32] A. Bartkiewicz, M. Szymczak, H. I. Van Langevelde et al., "The diversity of methanol maser mor. phologies from VLBI observations", 2009, A\&A 502155

(Parallaxes and proper motions of interstellar masers toward the Cygnus X star-forming complex: I. Membership of the Cygnus X region", 2012, A\&A 539 79

[34] C. Surcis, W. H. T. Vlemmings, H. J. van Langevelde et al., "EVN observations of 6.7 CHz methanol maser polarization in massive star-forming regions", 2013, A\&A 55673

35] M. A. Perez-Torres, C. Romero-Cañizales, A. Alberdi, and A. Polatidis, "An extremely prolific supernova factory in the buried nucleus of the starburst galaxy IC 694", 2009, A\&A 507 L17

[36] C. Fanti, R. Fanti, P. Parma et al., "Compact Steep Spectrum 3 CR radio sources - VLBl observations at $18 \mathrm{CM}^{\prime \prime}, 1985$, A\&A 143292

[37] R. Morganti, J. Fogasy, Z. Paragi et al., "Radio Jets Clearing the Way Through a Calaxy: Watching Feedback in Action", 2013, Science 3411082

[38] O. Witasse, J.P. Lebreton, M. K. Bird et al., "Overview of the coordinated ground-based observaA. Barthich

政 emission around a young high-mass star", 2005, A\&A 442 L61 
[40] Z. Paragi, G. B. Taylor, C. Kouveliotou et al., "A mildly relativistic radio jet from the otherwise normal type Ic supernova 2007gr", 2010, Nature 463516

[41] C. Spingola, J. P. McKean, M. W. Auger, "SHARP - V. Modelling gravitationally-lensed radio arcs imaged with global VLBI observations", 2018, MNRAS 4784816

[42] B. Marcote, Z. Paragi, J. W. T. Hessels et al., "The Repeating Fast Radio Burst FRB 121102 as Seen on Milliarcsecond Angular Scales", 2017, Ap) 834 L8

[43] M. K. Bird, M. Allison, S. W. Asmar et al., "The vertical profile of winds on Titan", 2005, Nature 438 800 